2008-01-01

\title{
Influence of Fiber Manufacturing Tolerances on the Spectral Response of a Bend Loss Based All-fiber Edge Filter
}

\author{
Pengfei Wang \\ Technological University Dublin, pwang@tudublin.ie \\ Gerald Farrell \\ Technological University Dublin, gerald.farrell@tudublin.ie \\ Yuliya Semenova \\ Technological University Dublin, yuliya.semenova@tudublin.ie
}

See next page for additional authors

Follow this and additional works at: https://arrow.tudublin.ie/engscheceart

Part of the Electrical and Computer Engineering Commons

\section{Recommended Citation doi:10.1364/A0.47.002921 \\ This Article is brought to you for free and open access by the School of Electrical and Electronic Engineering at ARROW@TU Dublin. It has been accepted for inclusion in Articles by an authorized administrator of ARROW@TU Dublin. For more information, please contact arrow.admin@tudublin.ie, aisling.coyne@tudublin.ie, gerard.connolly@tudublin.ie. Funder: Enterprise Ireland}

Wang, P., Farrell, G., Semenova, Y., Rajan, G.: Influence of fiber manufacturing tolerances on the spectral response of a bend loss based all-fiber edge filter. Applied Optics, Vol.47, 16, 2008, pp.2921-2925.

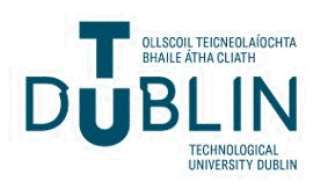




\section{Authors}

Pengfei Wang, Gerald Farrell, Yuliya Semenova, and Ginu Rajan

This article is available at ARROW@TU Dublin: https://arrow.tudublin.ie/engscheceart/40 


\title{
Influence of fiber manufacturing tolerances on the spectral response of a bend loss based all-fiber edge filter
}

\author{
Pengfei Wang, Gerald Farrell, Yuliya Semenova, Ginu Rajan \\ Applied Optoelectronics Center, School of Electronics and Communication \\ Engineering, Dublin Institute of Technology, Kevin Street, Dublin 8, Ireland \\ *Corresponding author:pengfei.wang@student.dit.ie
}

\begin{abstract}
It is shown that manufacturing tolerances of the fiber parameters bend radius and NA significantly influence the fiber bend loss performance and spectral response of a fiber-based edge filter. A theoretical model, validated by experimental results, is used to determine the changes in key spectral parameters for an edge filter, resulting from changes within their manufacturing tolerance range, for both the bend radius and NA. Finally is shown that bend-radius tuning during fabrication of such filters is a means of mitigating the effect of manufacturing variations.
\end{abstract}

Keywords: Singlemode fiber, manufacturing tolerance, bend loss, edge filter OCIS codes: $120.4820,120.4570,060.2310$ 


\section{Introduction}

Fiber bend loss has been previously investigated for application in areas such as fiber-optic sensing and communications [1-3]. Recently, it was shown that fiber bend loss could be used as the basis of an edge filter for an all-fiber ratiometric wavelength measurement system [4-7]. Based on the previously published work for fiber bend loss [8-10], both theoretical and experimental results have shown that both the bend radius and fiber length have a significant influence on the fiber bend loss $[6,7]$.

In addition, in practice, the fiber bend loss will be sensitive to the fiber's own parameters, such as fiber core radius, refractive index and so on. Given the manufacturing tolerances for singlemode fiber, small variations in fiber parameters are unavoidable. All of these parameter tolerances resulting from the manufacturing process may significantly affect the resulting ratio response of a fiber based ratiometric wavelength measurement system. Therefore, it is highly desirable to investigate the influence of these fiber parameter variations on the response of fiber bend loss based edge filters.

In past work, both fiber bend loss theoretical modeling and experiments were carried out on SMF28 fiber with a multi-turn structure and 1060XP fiber with a compact single-turn structure, e.g., bend radius of $11 \mathrm{~mm}$ with 22 turns in Ref. [6], and bend radius in $10.5 \mathrm{~mm}$ with single turn in Ref. [7]. In order to evaluate the fiber bend loss performance of a fiber edge filter employed in a wavelength measurement system, two important parameters, baseline loss and discrimination range have been also defined $[6,7]$. 
In this paper, the dependences of fiber bend loss on parameter tolerances are presented for the fiber core radius and the numerical aperture (NA) value. Through examination of the dependence of bend loss behavior on the parameters of bend loss sensitive singlemode fiber-1060XP, it is found that these small variations in these parameters have a significant impact on the spectral response of the fiber bend loss edge filter. We also show that bend-radius tuning during fabrication of such filters is a means of mitigating the effect of manufacturing variations.

\section{Design of a 1060XP based fiber edge filter}

Fig. 1 (a) shows a fiber edge filter used within a ratiometric wavelength measurement system. The input signal is split into two equal signals. One passes through the fiber bend loss edge filter (upper arm) and the other passes through a reference arm (lower arm). Photodiodes are placed at the ends of both arms. By measuring the ratio of the electrical outputs of the two photodiodes, we can determine the wavelength of the input signal assuming a suitable calibration has taken place. The fiber bend loss edge filter, for a measurable wavelength range from $\lambda_{1}$ to $\lambda_{2}$, provides a strong monotonically increasing wavelength dependent attenuation (discrimination range), from a given lower start wavelength (baseline loss, $\lambda_{1}$ ) to an end wavelength $\lambda_{2}$ [see Fig. 1(b)].

In our previously published work on wavelength ratiometric measurements $[4,5]$, it was found that with higher slope values for the transmission response of the edge filters for a given measurable wavelength range, the output ratio $R$, which was 
measured by a dual channel power meter, diverged from the actual transmission response of the edge filters at the upper end of the wavelength range due to the limited Signal-to-Noise Ratio (SNR) of real optical sources. To take account of this fact the slope of the transmission response of edge filters must be limited, effectively placing an upper limit on the useable discrimination. In regard to baseline loss, there are inevitable transmission losses, splicing losses, and insertion losses in such a system and these losses increase the baseline loss as well, reducing the overall signal power available for Optical-to-Electrical (OE) conversion and thus degrading accuracy due to noise. Ideally, the baseline loss should be lower than $5 \mathrm{~dB}$ and the discrimination range should be lower than $20 \mathrm{~dB}$ and larger than $15 \mathrm{~dB}$ for wavelength measurement.

For the 1060XP fiber, the essential parameters are shown in Table 1:

Fig. 2 shows that the calculated baseline loss (solid line), discrimination range (dashed line), measured baseline loss (hollow circles), discrimination range (solid squares) as a function of bend radius for the bend loss sensitive singlemode fiber-1060XP, with an applied absorbing layer and a fiber bend of 1 turn. A so-called effective bend radius (correction factor, 1.308@1500nm; 1.336@1600nm) is also employed in the theoretical modeling, as presented in Ref. [7]. In the experiments, a tunable laser is employed as a power source, which has an output power of $0 \mathrm{dBm}$ with a wavelength tuning range from 1500 to $1600 \mathrm{~nm}$. To experimentally determine the baseline loss and discrimination range, the bend losses of bare 1060XP fiber with an absorbing layer (to remove the reflection occurring at the interface between the cladding layer and air) were measured at $1500 \mathrm{~nm}$ and at $1600 \mathrm{~nm}$. The measurements 
were taken at $0.5 \mathrm{~mm}$ intervals over a bending radius range from 8.5 to $14 \mathrm{~mm}$.

In Fig.2, the theoretical modeling shows a reasonable agreement with the experimental results overall, and the divergence between the experimental and theoretical results is most likely caused by: 1) measurement error of the bending loss and errors due to temperature induced fluctuations of the bend loss; 2) the experimental inaccuracy of the bend radius (both baseline loss and discrimination range are sensitive to the bend radius as shown in Fig. 2; 3) the limitations of the absorbing layer material coated on the bare fiber cladding surface leading to partial reflections from the fiber cladding-air boundary so that radiation launched from fiber core will re-couple with the fundamental propagation mode in the fiber core, resulting in a perceptible quasi-periodic behaviour for the measured results in Fig. 2. Finally the calculation of the bend loss itself has some minor limitations: 1) the approximations made in the calculation from D. Marcuse [8] and 2) the calculation of bend loss is based on a scalar approximation to enable reasonable processing times with limited processing power and memory.

From Fig. 2, one can also see that both the baseline loss and discrimination range are highly sensitive to the bend radius. When the bend radius is less than $8 \mathrm{~mm}$, as in our previous experiments [11], it is found that when the bare fiber is bent, it is more susceptible to breakage. Furthermore, to reduce the fiber bend induced mechanical stress and improve the reliability of the fiber filter, it is suggested that a bend radius is larger than $8 \mathrm{~mm}$ is employed for fiber edge filter applications. Thus in this paper a bend radius of $10.5 \mathrm{~mm}$ is chosen to characterize the influence of bend loss due to the 
manufacturing tolerance of fiber parameters.

\section{Influence of the manufacturing tolerance of key fiber parameters on the performance of a fiber-based edge filter}

For a 1060XP fiber based edge filter, the influence of manufacturing variations in key fiber parameters needs to be considered in the design process. In the first instance the influence of variations in the fiber core radius (nominal value $2.65 \mu \mathrm{m}$ ) was modeled for a range of core radii from 2.35 to $2.95 \mu \mathrm{m}$. The results are shown in Fig. 3 in which the calculated baseline loss (solid square line) is shown along with the discrimination range (hollow square line) as a function of fiber core radius for 1060XP fiber with an absorbing layer. The fiber bend radius is $10.5 \mathrm{~mm}$, and bend length is 1 turn.

It is clear that both the baseline loss and discrimination range, decrease rapidly with an increase in fiber core radius. In practice the manufacturing tolerance for the fiber core radius is $\pm 0.25 \mu \mathrm{m}$, so from Fig. 3, one can see that a large variation in the baseline loss and discrimination range will occur within this manufacturing tolerance range. The worst case variation for either the baseline loss value or the discrimination range value is the difference between the highest value, calculated for the lowest possible core radius $(2.4 \mu \mathrm{m})$ and the lowest value calculated for the highest possible core radius $(2.9 \mu \mathrm{m})$. For the baseline loss the worst case variation is $34.6 \mathrm{~dB}$, and for the discrimination range it is $65.3 \mathrm{~dB}$. 
Next, the influence of manufacturing tolerance for fiber NA value was modelled. During the fiber manufacturing process, the tolerance of refractive index difference between fiber core and cladding can be controlled below $\pm 10 \%$, and the corresponding calculated core NA tolerance (nominal value 0.1393 ) of the 1060XP fiber is thus within the range from 0.1321 to 0.1461 . The results of the modeling are shown in Fig. 4, for a range of NA value from 0.1275 to 0.1515 .

The baseline loss and discrimination range vary with the fiber NA, and for a fiber with lower NA value the result is a larger baseline loss and discrimination range. Within the manufacturing tolerance range for the NA, the worst case variation for the baseline loss is $27.6 \mathrm{~dB}$, and for the discrimination range is $52.3 \mathrm{~dB}$.

\section{Discussion}

Even allowing for worst case nature of manufacturing tolerances, for the fiber parameters core radius and NA it is clear from Figures 3 and 4 that such variations have a profound effect on the discrimination range and baseline loss of a fiber bend loss edge filter. In manufacturing such filters in significant quantities such variations in discrimination range and baseline loss would result in unacceptably low production yields. However in practice there are mitigating issues which can reduce or even eliminate the impact of manufacturing tolerances.

The first issue is the statistical nature of manufacturing tolerances. It is known for example that the core radius variations of a fiber normally conform to a Gaussian statistical distribution, which has been demonstrated in the Ref. [12]. To determine the standard deviation it can be assumed that the worst case variations about the 
nominal value of the core radius correspond to approximately \pm 3 standard deviations about the nominal value. Based on Gaussian statistics one could assume the while the worst case variation is indeed $\pm 0.25 \mu \mathrm{m}$, in practice in $95 \%$ of cases the actual fiber tolerance will be within \pm 2 standard deviations or $\pm 0.166 \mu \mathrm{m}$. The corresponding variation of the discrimination range is reduced from $65.32 \mathrm{~dB}$ worst case to $39.55 \mathrm{~dB}$ for \pm two standard deviations, reducing the impact of manufacturing variations.

The second mitigating issue is that fiber bend loss filters can be fabricated to allow the discrimination range and baseline loss to be set and fixed at the point of manufacture. This means that a preset mandrel around which the fiber is fixed cannot be used but instead a freespace fiber bend loop is fabricated and the radius of the loop is "tuned" manually to give the correct discrimination at a given wavelength. The fiber loop is then fixed using an epoxy droplet at the crossover point of the fibers. To verify if this is possible the model described earlier is used to determine the fiber bend radius that would need to be set to ensure that the discrimination range is equal to the design example value of $16.32 \mathrm{~dB}$ (achieved with a nominal core radius $(2.65 \mu \mathrm{m})$ for a bend radius of $10.5 \mathrm{~mm})$ where the actual core radius can vary by $\pm 0.166 \mu \mathrm{m}( \pm 2$ standard deviations). The results are presented in Table 2. As expected for low values of core radius, a larger bend radius is needed, up to $14.55 \mathrm{~mm}$. For high values of core radius a smaller bend radius is needed, down to $8 \mathrm{~mm}$, which is acceptable in terms of the minimum radius needed to avoid fiber failure.

Variations in the fiber NA from nominal can be also be dealt with using bend radius tuning during fabrication. The nominal NA is 0.1393. Again assuming 
Gaussian statistics, the worst case value of NA variation is equal to approximately three standard deviations. For $95 \%$ of fibers the variation will be within \pm two standard deviations or \pm 0.004887 . If bend radius tuning is employed to ensure that even with NA variations the discrimination range is still $16.32 \mathrm{~dB}$, then the bend radius range needed to compensate NA variations is shown in Table 3.

The bend radius needed to compensate for NA variations has a minimum value of $7.25 \mu \mathrm{m}$, which is acceptable in terms of fiber reliability. Bend radius "tuning" during fabrication of the fiber filter is thus a feasible approach to reducing the effect of manufacturing tolerances. To confirm this, for a design bend radius of $10.5 \mathrm{~mm}$, we calculated the tuned bend radius required to meet the edge filter specifications over the range of possible fiber core radii and NA values. The results are shown in Fig. 5 which shows in a graphical form that is possible to compensate for manufacturing variations in the fiber core size and fiber NA changes, by tuning the actual bend radius used.

In practice the need to "tune" the bend radius to meet a given discrimination range should not be an onerous task. The fiber to be used for a batch of fiber bend loss filters will most likely come from the same drawn length of fiber, so similar "tuned" bend radii can be expected for a given batch of filters. Finally it is also true that for many applications of such fiber bend loss filters (such as wavelength measurement as discussed earlier), there is no need to achieve a precise discrimination through radius tuning as in any event the overall system calibration will take account of variations in the discrimination range. 
Finally it is recognised that parameter variations during manufacture will not occur in just one parameter at a time, but rather variations in several parameters will occur. We have not considered such combined variations in this paper. However we have shown that in the fabrication of bend-loss based fiber edge filters, bend radius tuning can be employed to mitigate the effects of parameter variations. Where the fiber used displays variations from a nominal value for several parameters, bend radius tuning will still offer a means of achieving the correct filter response, albeit with a lower yield, depending on the magnitude of the parameter variations.

\section{Conclusions}

The design of a 1060XP singlemode fiber based edge filter has been examined in the context of variations in fiber parameters. We have shown that two such parameters, core radius and NA, influence the fiber bend loss spectral response performance significantly, and two analytical models have been presented for calculating the influence of the manufacturing tolerance of the fiber parameters on fiber bend loss. Finally we have shown that bend-radius tuning during the fabrication of such filters is a viable way to mitigate the effect of manufacturing variations.

\section{References}

1. M. T. Wlodarczyk, "Wavelength referencing in single-mode microbend sensors," Opt. Lett., 12, 741-743 (1987)

2. S. H. Nam, and S. Yin, "High-temperature sensing using whispering gallery mode resonance in bent optical fibers," IEEE Photon. Technol. Lett., 17, 2391-2393 (2005). 
3. M. D. Nielsen, N. A. Mortensen, M. Albertsen, J. R. Folkenberg, A. Bjarklev, and D. Bonacinni, "Predicting macrobending loss for large-mode area photonic crystal fibers," Opt. Express, 12, 1775-1779 (2004).

4. Q. Wang, G. Farrell and T. Freir, "Study of transmission response of edge filters employed in wavelength measurements," Appl. Opt., 44, 7789-7792 (2005).

5. Q. Wang, G. Rajan, P. Wang and G. Farrell, "Resolution investigation of ratiometric wavelength measurement system," Appl. Opt., 46, 6362-6367 (2007).

6. Q. Wang, G. Farrell, T. Freir, G. Rajan and P. Wang, "Low-cost wavelength measurement based on a macrobending single-mode fiber," Opt. Lett., 31, 1785-1787 (2006).

7. P. Wang, G. Farrell, Q. Wang and G. Rajan, "An optimized macrobending-fiber-based edge filter," IEEE Photon. Technol. Lett., 19, 1136-1138 (2007).

8. D. Marcuse, "Curvature loss formula for optical fibers," J. Opt. Soc. Am., 66, 216-220, (1976).

9. H. Renner, "Bending losses of coated single-mode fibers: a simple approach," J. Lightwave Technol., 10, 544-551 (1992).

10. L. Faustini and G. Martini, "Bend loss in single-mode fibers," J. Lightwave Technol., 15, 671-679 (1997).

11. P. Wang, Q. Wang, G. Farrell, G. Rajan, T. Freir and J. Cassidy, "Investigation of macrobending losses of standard single mode fiber with small bend radii," Microw. Opt. Techn. Lett. , 49, 2133-2138 (2007).

12. P. H. Krawarik and L. S. Watkins, "Fiber geometry specifications and its relation to measured fiber statistics,” Appl. Opt., 17, 3984-3989 (1978). 


\section{Figure captions}

Fig.1. (a) Schematic configuration of the ratiometric wavelength measurement system with the fiber edge filter; (b) desired spectral response of the fiber edge filter.

Fig.2. Calculated and measured baseline transmission loss and discrimination range as a function of bend radius for 1060XP fiber with an absorbing layer, the fiber length is 1 turn.

Fig.3. Calculated baseline transmission loss and discrimination range as a function of variable fiber core radius for 1060XP fiber with an absorbing layer, the fiber bend radius is $10.5 \mathrm{~mm}$, and length is 1 turn.

Fig.4. Calculated baseline transmission loss and discrimination range as a function of fiber core NA for 1060XP fiber with an absorbing layer, the fiber bend radius is $10.5 \mathrm{~mm}$, and length is 1 turn.

Fig. 5 Calculated results for the changes in the fiber core and NA as a function of the required fiber bending radius.

A axis: "Range of possible fiber core radius values based on manufacturing tolerance $(\mu \mathrm{m})$ "

B axis: "Range of possible NA values based on manufacturing tolerance" 


\section{Tables}

Table 1. Parameters of the 1060XP singlemode fiber; (the refractive index values are defined at a wavelength of $1550 \mathrm{~nm})$.

Table 2. Fiber bend radius range needed to compensate for two-standard deviation variations in the fiber core radius.

Table 3. Fiber bend radius range needed to compensate for two-standard deviation variations in the fiber NA. 


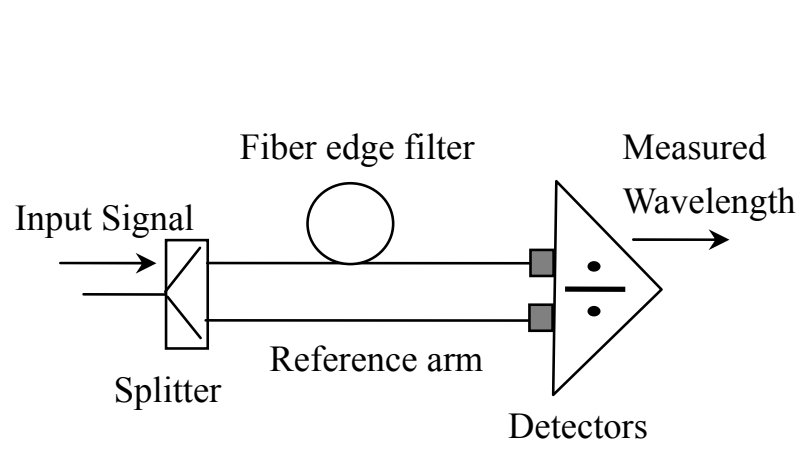

(a)

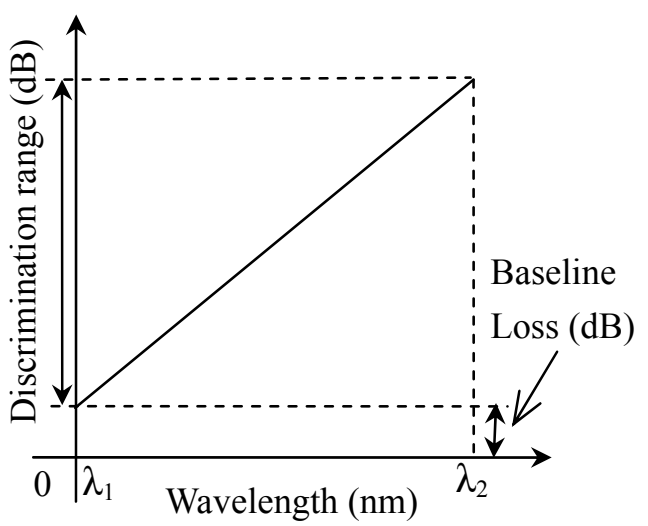

(b)

Fig.1. (a) Schematic configuration of the ratiometric wavelength measurement system with the fiber edge filter; (b) desired spectral response of the fiber edge filter. 


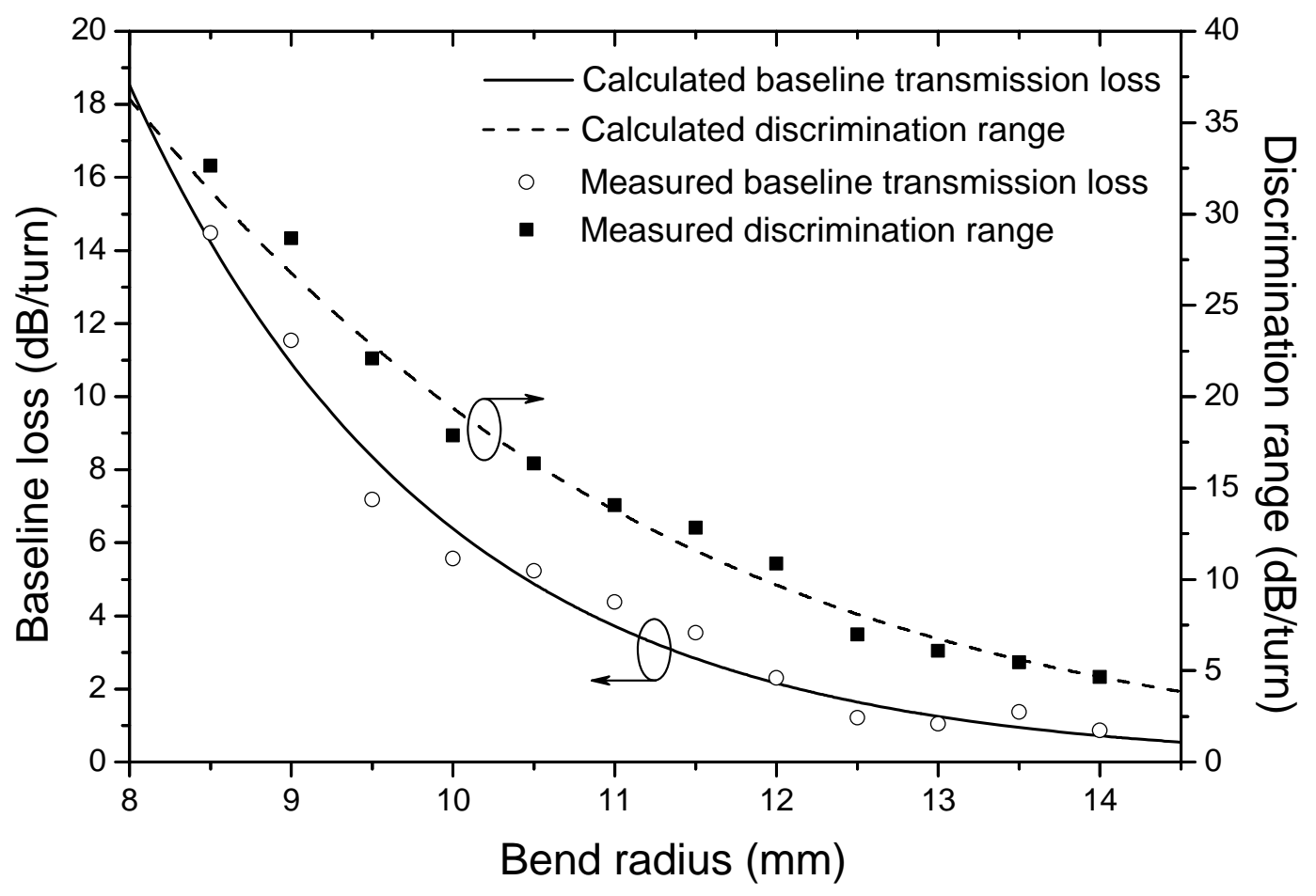

Fig.2. Calculated and measured baseline transmission loss and discrimination range as a function of bend radius for 1060XP fiber with an absorbing layer, the fiber length is 1 turn. 


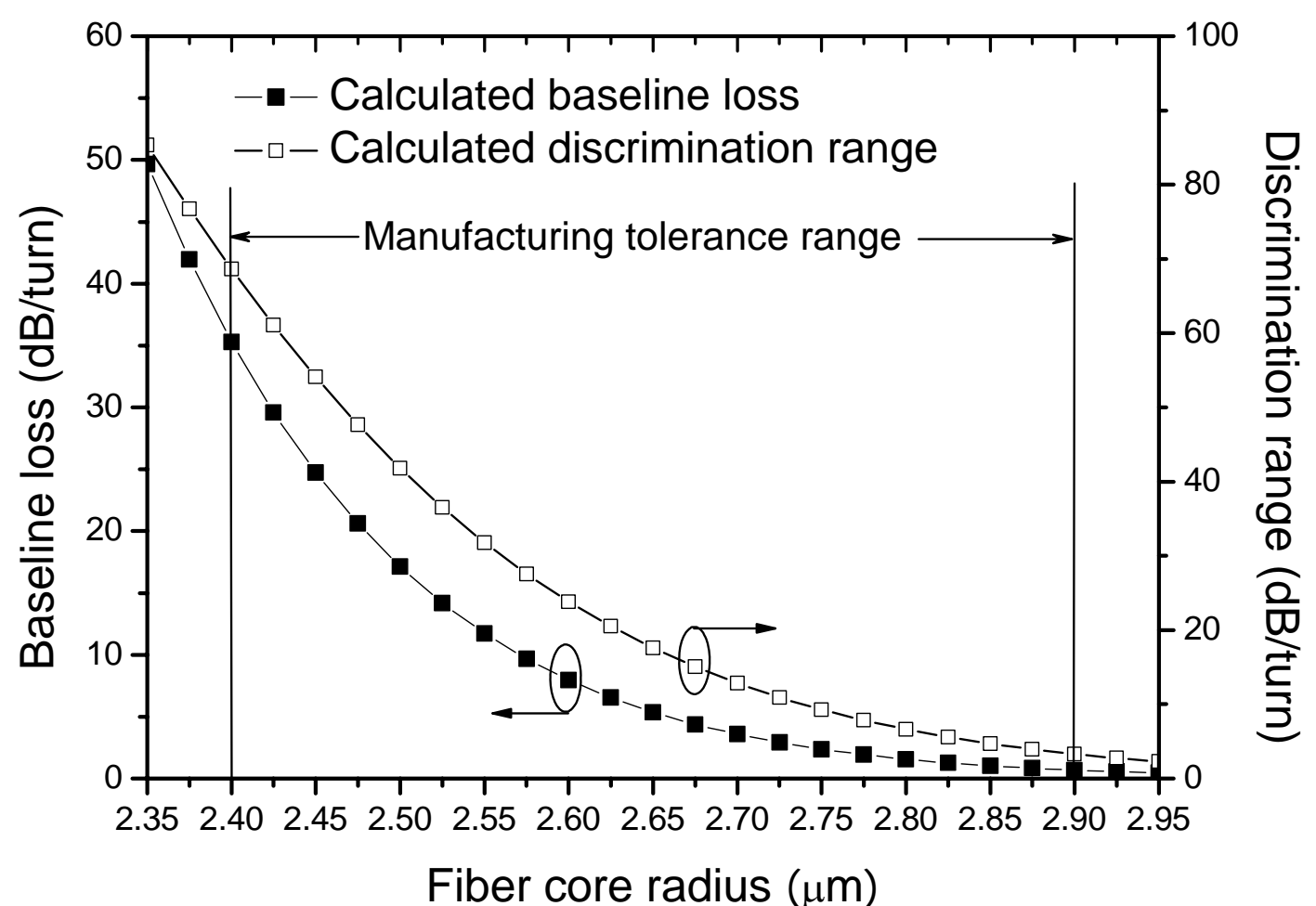

Fig.3. Calculated baseline transmission loss and discrimination range as a function of variable fiber core radius for 1060XP fiber with an absorbing layer, the fiber bend radius is 10.5 $\mathrm{mm}$, and length is 1 turn. 


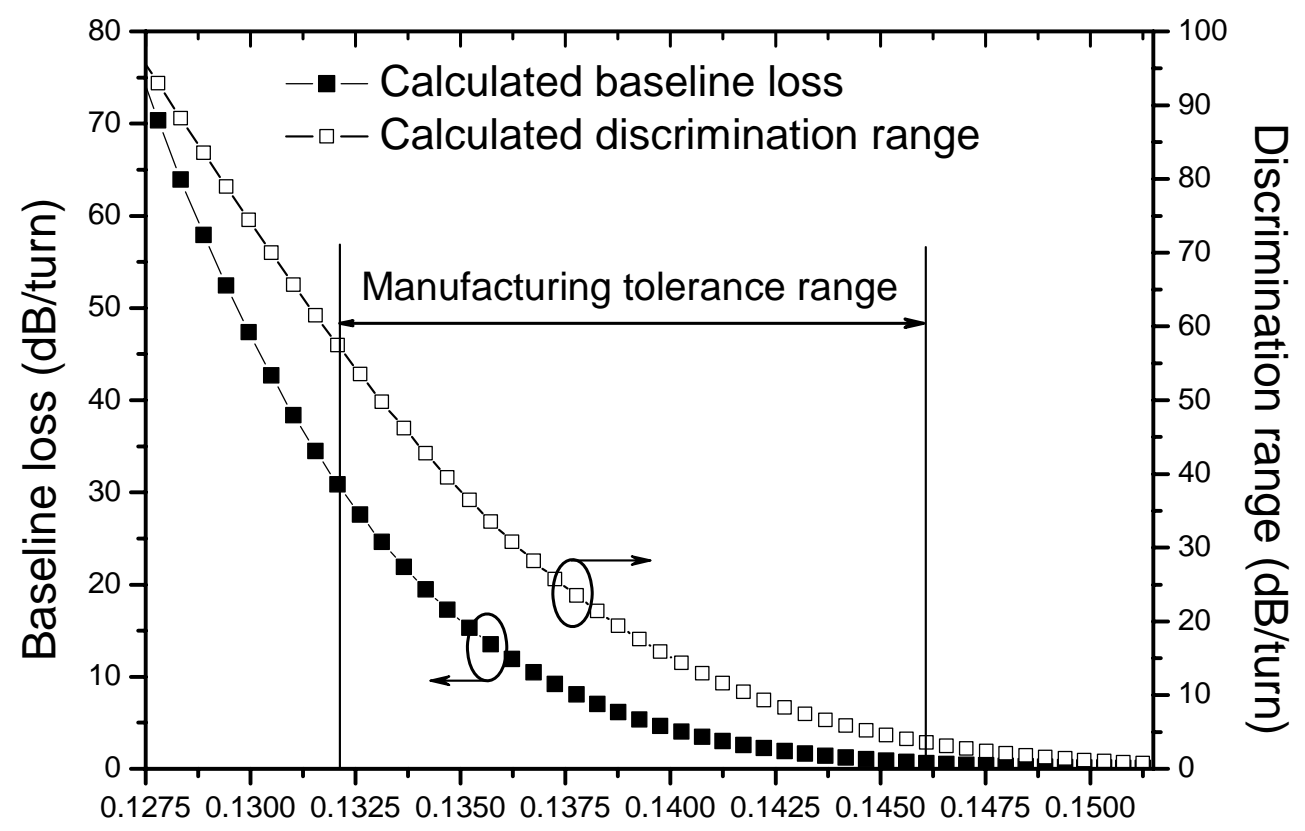

Fiber NA

Fig.4. Calculated baseline transmission loss and discrimination range as a function of fiber core NA for 1060XP fiber with an absorbing layer, the fiber bend radius is $10.5 \mathrm{~mm}$, and length is 1 turn. 


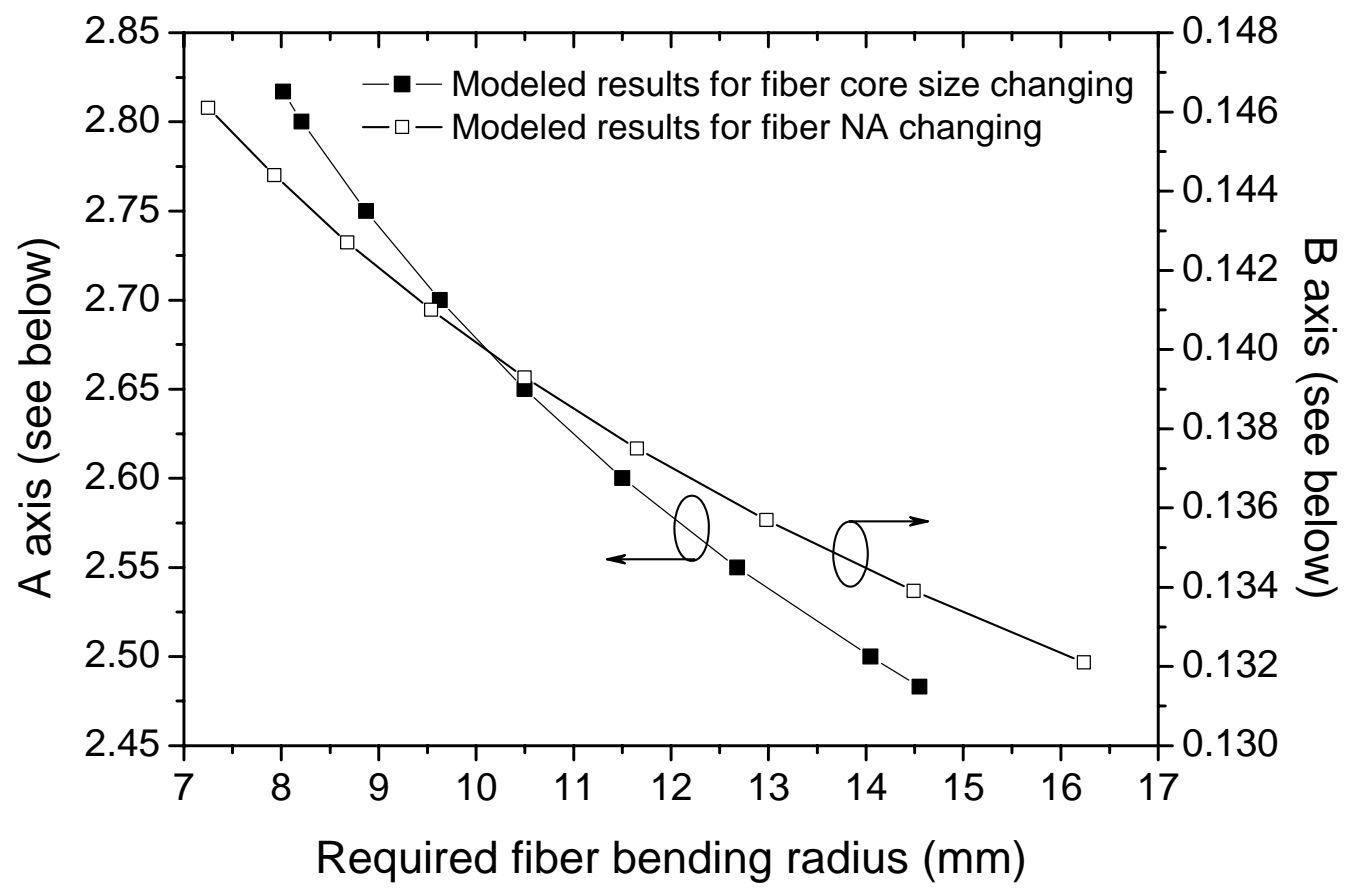

Fig. 5 Calculated results for the changes in the fiber core and NA as a function of the required fiber bending radius.

A axis: "Range of possible fiber core radius values based on manufacturing tolerance $(\mu \mathrm{m})$ " B axis: "Range of possible NA values based on manufacturing tolerance" 
Table 1. Parameters of the 1060XP singlemode fiber; (the refractive index values are defined at a wavelength of $1550 \mathrm{~nm}$ )

\begin{tabular}{cc}
\hline \hline Parameter of fiber & $1060 \mathrm{XP}$ \\
\hline Refractive index difference & $0.0067 \pm 10 \%$ \\
(between fiber core and cladding) & \\
Diameter of fiber core & $5.3 \pm 0.5 \mu \mathrm{m}$ \\
Diameter of fiber cladding & $125 \pm 0.5 \mu \mathrm{m}$ \\
Nominal core NA (Numerical Aperture) & 0.1393 \\
\hline \hline
\end{tabular}


Table 2. Fiber bend radius range needed to compensate for two-standard deviation variations in the fiber core radius

\begin{tabular}{ccc}
\hline \hline Fiber core radius $\mathrm{R}(\mu \mathrm{m})$ & $\Delta \mathrm{R}(\mu \mathrm{m})$ & Bend radius needed $(\mathrm{mm})$ \\
\hline 2.483 (lowest) & -0.166 & 14.55 \\
2.65 (nominal) & 0 & 10.50 \\
2.817 (highest) & +0.166 & 8.02 \\
\hline \hline
\end{tabular}


Table 3. Fiber bend radius range needed to compensate for two-standard deviation variations in the fiber NA

\begin{tabular}{ccc}
\hline \hline Fiber NA & $\Delta \mathrm{NA}$ & Bend radius needed $(\mathrm{mm})$ \\
\hline 0.1321 (lowest) & -0.0072 & 16.24 \\
0.1393 (nominal) & 0 & 10.50 \\
0.1461 (highest) & +0.0068 & 7.25 \\
\hline \hline
\end{tabular}

